\title{
¿Cómo llega el agua a las nubes? Construcción de explicaciones sobre cambios de estado en educación infantil
}

\author{
Sabela F. Monteira \\ Departamento de Didácticas Aplicadas, Facultade de Ciencias da Educación. Universidade de Santiago de \\ Compostela.Santiago de Compostela.España.sfmonteira@gmail.com \\ ORCID: https:// orcid.org/0000-0001-9115-298X \\ María Pilar Jiménez Aleixandre \\ Departamento de Didácticas Aplicadas, Facultade de Ciencias da Educación. Universidade de Santiago de \\ Compostela.Santiago de Compostela. España.marilarj.aleixandre@usc.es \\ ORCID: http:// orcid.org/0000-0002-2253-9651
}

[Recibido: 26 Diciembre 2017. Revisado: 11 Marzo 2018. Aceptado: 19 Diciembre 2018]

\begin{abstract}
Resumen: Este estudio de caso en un aula de tercero de educación infantil (23 niñas y niños, 5-6 años, y su maestra) examina la evolución de las explicaciones del alumnado sobre cambios de estado líquido-gas a lo largo de cinco meses de un proyecto escolar de ciencias. Para construir sus explicaciones movilizaron tanto su conocimiento cotidiano como el conocimiento científico escolar. Desarrollaron la capacidad de aplicar el vocabulario científico para explicar sus experiencias cotidianas. El fenómeno de evaporación fue identificado y explicado por el alumnado con mayor facilidad que el fenómeno de condensación. Proponemos una rúbrica para analizar la construcción de explicaciones. Las implicaciones educativas sugieren que es recomendable iniciar el estudio de los cambios de estado en educación infantil, ya que el alumnado reconoce estos fenómenos, los relaciona con su conocimiento cotidiano y es capaz de construir explicaciones que pueden servir de base para desarrollar otras más complejas.
\end{abstract}

Palabras clave: Educación infantil; Explicaciones; Cambios de Estado.; Experimentación.

How does water get to the clouds? Building explanations about state changes in Early Childhood Education

Abstract: This case study in a third year Early Childhood Education (ECE) classroom (23 children, 5-6 years old) and their teacher examines the evolution in children's explanations about state changes between liquid and gas in the course of a school science project that lasted for five months. They mobilized both their everyday and school science knowledge in order to build explanations. They developed the ability to apply scientific vocabulary to account for their everyday experiences. Children identified the phenomenon of evaporation more easily than the condensation. We propose a rubric for the analysis of the construction of explanations. Educational implications suggest that state changes should be addressed from ECE, since children are able to identify these phenomena, relate them to their everyday knowledge and build explanations about them, which may serve as a basis for developing more complex ones.

Keywords: Early Childhood Education; Explanations; State Changes; Experimentation.

Para citar este artículo: Monteira, S. F., Jiménez Aleixandre, M. P., (2019) ¿Cómo llega el agua a las nubes? Construcción de explicaciones sobre cambios de estado en educación infantil. Revista Eureka sobre Enseñanza y Divulgación de las Ciencias 16(2), 2101. doi: 10.25267/Rev_Eureka_ensen_divulg_cienc.2019.v16.i2.2101

\section{Introducción}

Pese a que la participación en ciencias desde educación infantil beneficia el rendimiento posterior del alumnado en este campo (OCDE, 2012), hay un escaso número de las investigaciones y publicaciones en Didáctica de las Ciencias Experimentales sobre este nivel educativo. Estos estudios apuntan a los beneficios de un currículo innovador (Leuchter, Saalbach y Hardy, 2014) y guiado por los intereses del alumnado (Siry y Max, 2013).

\footnotetext{
Revista Eureka sobre Enseñanza y Divulgación de las Ciencias

Universidad de Cádi : APAC-Eureka. ISSN: 1697-011X

bttp:/ / dx.doi.org/10.25267/Rev_Eureka_ensen_divulg_cienc.2019.v16.i2.2101

http:// reuredc.uca.es
} 
Nuestro estudio se sitúa en una perspectiva que considera la Ciencia como un conjunto de prácticas, y que aprender ciencias implica participar en las prácticas de la disciplina (Osborne 2014). Una de estas prácticas científicas es la construcción de explicaciones (National Research Council (NRC), 2012), que además es identificada como una de las tres grandes dimensiones que conforman la competencia científica, junto con la evaluación y diseño de investigaciones científicas y la interpretación de datos y pruebas (Organización para la Cooperación Económica y el Desarrollo, OCDE, 2016). La pregunta de investigación es:

¿Cómo evolucionan, en cuanto a la identificación de componentes y procesos y al establecimiento de relaciones, las explicaciones sobre los cambios de estado líquido-gaslíquido del alumnado de tercer curso de educación infantil (5-6 años), en particular en el contexto de la realización y discusión reflexiva de experimentos?

\section{Construcción de explicaciones}

Una explicación es, según McNeill (2011), «an account of how or why a phenomenon occurs and explaining why the natural world works in particular ways» (p. 795). Las explicaciones causales sobre fenómenos naturales son un tipo de enunciado de conocimiento relevante en ciencias (Jiménez Aleixandre, 2010). Aunque construir explicaciones favorece el aprendizaje de las ciencias (McNeill y Krajcik, 2009; Songer y Gotwals, 2012), con frecuecia los alumnos reciben muchas explicaciones, pero no se les solicita construirlas (Zangori, Forbes y Biggers, 2013).

Hadzigeorgiou (2015) apunta que los niños construyen explicaciones a partir de sus experiencias cotidianas. Fleer y Pramling (2015) consideran que los conceptos cotidianos, construidos a partir de las experiencias del día a día, son centrales para el desarrollo de los conceptos científicos. Vygotsky (1978) sugirió que el desarrollo de ambos tipos de conceptos debe entenderse como un proceso unificado.

La interacción con compañeras y compañeros juega un papel destacado en la construcción colectiva de conceptos científicos en edades tempranas (Siry y Kremer, 2011). Mercer (2000) y Littleton y Mercer (2013) discuten la utilización de las conversaciones como una herramienta de aprendizaje. Según Mercer las conversaciones en el aula pueden ser de disputa, acumulativas o exploratorias, siendo estas últimas las más productivas en el aprendizaje colectivo.

Songer y Gotwals (2012) emplean la perspectiva de las progresiones de aprendizaje (LP, learning progressions) en su análisis sobre la construcción de explicaciones por el alumnado de primaria con cada vez menor andamiaje de su profesora. En nuestro estudio, nos interesa la noción de LP como trayectorias en el aprendizaje de conceptos o en la participación en prácticas científicas que comprenden varios pasos de menor a mayor sofisticación (Berland y McNeill, 2010). Estos pasos son descritos en base al análisis de los desempeños del alumnado, no a la lógica de la disciplina. Están comprendidos entre un extremo inferior, con el que comienza el alumnado; y un nivel superior, al que se pretende que llegue. Conocer cómo evolucionan los desempeños el alumnado facilita el diseño de secuencias didácticas adaptadas a sus capacidades.

\section{La comprensión de los cambios de estado}

La comprensión del alumnado de diferentes edades sobre los cambios de estado ha sido estudiada en el contexto del aula (Johnson 1998; Tytler 2000; Tytler y Peterson, 2004) y mediante entrevistas (Osborne y Cosgrove, 1983). Explicarlos requiere comprender la naturaleza de la materia y establecer relaciones con variables como la temperatura. Todos los 
estudios consultados muestran que muchas de las explicaciones del alumnado difieren de las científicas.

Orborne y Cosgrove (1983) muestran que una idea recurrente del alumnado de secundaria es que el vapor se convierte en aire cuando deja de ser visible. Es decir, existe una dificultad para comprender el proceso de evaporación y la presencia de agua en el aire. Según Johnson (1998), que el alumnado apropie la noción de que la materia está formada por partículas facilita la comprensión de este proceso.

Los resultados del estudio de Tytler (2000) con alumnado de primero y sexto curso de educación primaria muestran que su capacidad de establecer más relaciones en sus explicaciones sobre evaporación que sobre condensación. Ninguno de los participantes relacionó los fenómenos de condensación con la formación de nubes, o con la lluvia, ni tampoco emplearon la palabra "condensación". Sin embargo, alumnos de ambos niveles usaron la palabra "evaporación" y establecieron relaciones entre este fenómeno y la formación de nubes. También el estudio de Cruz-Guzmán, García-Carmona y Criado (2017) con alumnado de educación infantil indica que la condensación resulta más difícil de entender que la de la evaporación. Para ambos fenómenos, la comprensión depende del contexto, especialmente en el caso de los niños más pequeños (Tytler y Peterson, 2004).

En definitiva, pese a que se trata de procesos que el alumnado experimenta en su día a día, explicarlos de manera científica es exigente, especialmente para niños y niñas pequeños.

\section{Diseño de la investigación}

El estudio está enmarcado en una perspectiva cualitativa y el diseño es un estudio de caso longitudinal (Merriam, 2009). Acompañamos a un grupo durante los tres años de educación infantil. Este trabajo se centra en datos del tercer año del estudio, en el que se grabaron en video 24 sesiones (27,5 horas). Nos centramos en el análisis del discurso del alumnado, ya que el foco es la construcción de explicaciones sobre los cambios de estado. Por ello, los datos examinados en profundidad son las transcripciones de las sesiones. Los participantes se identifican con seudónimos. El alumnado se mueve por la clase y en ocasiones no está en el punto de enfoque de la cámara, por lo que no siempre es posible identificar al niño o niña que interviene.

\section{Participantes y contexto}

Los participantes son 13 niños, 10 niñas (5-6 años) y su maestra. Realizaron durante cinco meses un proyecto sobre nubes. Un niño y una niña, que asistían a actividades de ciencias extraescolares, propusieron que «las nubes están hechas de agua», y el grupo comenzó a discutir cómo podría llegar el agua a las nubes. La maestra tomó las preguntas de los niños como punto de partida para estudiar los cambios de estado del agua.

Seis sesiones (Tabla 1) son examinadas en profundidad, como se justifica en el apartado métodos de análisis. 
Tabla 1. Contenidos de las sesiones

\begin{tabular}{|c|c|}
\hline Sesión & Contenidos: temas y acciones \\
\hline 5 & $\begin{array}{l}\text { Observación de nubes en el patio del colegio } \\
\text { Realización del experimento 1, "Evaporación" }\end{array}$ \\
\hline 6 & $\begin{array}{l}\text { Fenómenos meteorológicos: la helada } \\
\text { Control del experimento } 1 \\
\text { Realización del experimento 2, "Ebullición" }\end{array}$ \\
\hline 7 & $\begin{array}{l}\text { Cuento sobre nubes } \\
\text { Control del experimento } 1 \\
\text { Repetición del experimento } 2\end{array}$ \\
\hline 8 & $\begin{array}{l}\text { Repetición del experimento } 2 \\
\text { Representación gestual de los tres estados del agua } \\
\text { Dibujo del experimento } 2\end{array}$ \\
\hline 13 & Agua evaporada en el aire: empleo de dos analogías (maestra) \\
\hline 14 & $\begin{array}{l}\text { Discusión de una representación de las medidas del experimento } 1 \\
\text { Dibujo del experimento } 1\end{array}$ \\
\hline
\end{tabular}

En estas seis sesiones se realizan dos experimentos que implican cambios de estado gaslíquido, a temperatura ambiente y a temperatura de ebullición. Las fases para llevarlos a cabo, descritas a continuación, son: 1) introducción del material por la maestra; 2) discusión de propuestas: '¿qué podemos hacer con este material?'; 3) predicciones: '¿qué va a suceder?'; 4) realización y observación del experimento; y 5) puesta en común.

Experimento 1- Evaporación, sesión 5 y observaciones registradas durante tres semanas: 1) La maestra presenta el material: agua, cinta métrica, y vasos. 2) Varios niños indican que ese material es para hacer nubes. La maestra les pregunta cómo pueden hacer y los niños indican que dejando el agua en los vasos. La maestra les sugiere, además, marcar la altura del agua y poner un vaso dentro y uno fuera. 3) El alumnado indica que se formarán nubes: que medirán lo mismo que la altura del agua; que se formarán dentro de la clase. 1) La maestra presenta las bolsas herméticas. 2) El alumnado indica que también se deben llenar de agua y que 3) la 'nube' se formará dentro de la bolsa porque el agua no puede salir. 4) Durante tres semanas, el alumnado examina y registra cambios en la altura del agua de los vasos; y observa las gotas en las paredes internas de las bolsas. 5) Las observaciones son interpretadas mediante discusión colectiva.

Experimento 2 - Ebullición, sesiones 6, 7, y 8:1) La maestra presenta el material: jarra calienta aguas, agua y espejo. 2) El alumnado indica que el calienta aguas es un 'calienta bibes' para echar agua en él; la maestra les insiste en medir el agua que van a echar. 3) El alumnado indica que el agua se va a calentar y hacer burbujas al echarla allí. 4) Miden una cantidad de agua, enchufan el calienta aguas y observan el humo que sale. La maestra les indica entonces que sitúen el espejo encima del aparato y que lo toquen, de manera que pueden sentir el agua condensada. Comprueban que no queda agua en el aparato. 5) Las observaciones son interpretadas mediante discusión colectiva.

\section{Métodos de análisis}

Las grabaciones fueron transcritas, y el análisis del discurso y de las interacciones de los participantes (Gee, 2005; Kelly y Green, 2019; Mercer, 2000) se realizó sin emplear software. La reducción de datos, siguiendo las fases indicadas a continuación, y el análisis se llevaron a cabo individualmente por las autoras. Las categorías de análisis emergen de la interacción entre la literatura y los datos, en un proceso iterativo, en que las autoras realizan una inmersión profunda en los datos; se elabora una rúbrica provisional; se codifican las transcripciones, se 
modifica la rúbrica en los casos en que es necesario, y se procede a un nuevo análisis. Después de cada fase, los desacuerdos se resolvieron mediante discusión. Las fases, esquematizadas en la Figura 1, son las siguientes:

1. Análisis temático del contenido (Neuendorf, 2002) de las transcripciones, para identificar temas recurrentes en más de una sesión: cambios de estado (20 sesiones), formación de nubes (18), tipos de nubes (13), fenómenos atmosféricos (10) y ciclo del agua (5).

2. Distribución en episodios y participación individual: las 20 sesiones en las que se trabajó y discutió sobre los cambios de estado fueron examinadas mediante análisis de discurso (Gee, 2005) y divididas en episodios. Se identificaron 13 sesiones en las que se discutieron de forma sistemática cambios de estado. Caracterizamos como sistemática la discusión en profundidad de estos fenómenos, en oposición a simples menciones. Se registró el número de intervenciones de cada participante (Tabla 2).

Tabla 2. Participación en el discurso

\begin{tabular}{|l|c|c|}
\hline Participación (\% turnos rango) & \% total turnos alumnado & $\mathbf{N}^{\mathbf{o}}$ de alumnos (niñas/niños) \\
\hline Alta $(14,8-4,5 \%)$ & 41,4 & $6(0 / 6)$ \\
\hline Media $(4,4-1,7 \%)$ & 10,6 & $4(1 / 3)$ \\
\hline Baja $(\leq 1,6 \%)$ & 48 & $13(9 / 4)$ \\
\hline
\end{tabular}

3. Identificación de explicaciones: ya que pretendemos identificar una evolución en las explicaciones, de menor a mayor sofisticación, proponemos que la construcción de explicaciones implica tres pasos de complejidad creciente, el tercero inspirado en los niveles para "theoretical claims" propuestos por Kuhn y Pearsall (2000): 1) identificar componentes relevantes para el fenómeno; 2) identificar procesos relevantes para el fenómeno; y 3) establecer relaciones entre ellos. Por ello, se han analizado no sólo explicaciones, sino también los turnos en los que se refieren a componentes y procesos implicados en los cambios de estado. Se han categorizado como explicación los turnos en que se expresan relaciones, según los siguientes criterios:

1. Se describe cómo tiene lugar el fenómeno

2. Se expresa una relación causal entre componentes y procesos

3. Se aplica una explicación a un contexto diferente

En educación infantil es frecuente que el alumnado repita lo que oye. Estas repeticiones no fueron codificadas como una nueva explicación. En las 13 sesiones se identificaron 87 explicaciones sobre cambios de estado construidas por el alumnado.

4. Identificación de pautas de evolución: se examinó el contenido de las 87 explicaciones para identificar pautas de evolución, es decir, de qué manera a) el alumnado incorporó a sus explicaciones nuevas descripciones o b) relaciones causales, o c) las aplicó a nuevos contextos. Se trata de 47 episodios de las 6 sesiones que se discuten en profundidad, en los que el alumnado construyó 35 explicaciones.

La construcción de explicaciones en esta aula es colectiva, más que un producto individual, tiene lugar durante la discusión en grupo, guiada por la maestra y encaminada a interpretar y dar sentido a los fenómenos estudiados. Por este carácter colectivo, el criterio para considerar que una explicación es producida por los miembros de la clase que intervienen, es no sólo que fuera empleada una vez, sino que las explicaciones posteriores, o bien estuvieran basadas en ella o no entraran en contradicciones con ella. Esto corresponde al tipo de conversación exploratoria (Mercer, 2000). Mercer y colaboradores consideran exploratorio al diálogo en el que: los participantes escuchan activamente, formulan preguntas, comparten información relevante, las ideas pueden ser debatidas, las contribuciones se basan en lo que ha sucedido y 
se ha discutido antes, las ideas y opiniones son tratadas con respeto, hay un sentido de propósito compartido y el grupo busca acuerdo para las decisiones conjuntas.

5. Tipo de conocimientos movilizados: al realizar el análisis de contenido de las explicaciones, emergió el interés de analizar el tipo de conocimientos movilizados en ellas: cotidiano y científico escolar. Cotidiano es el que niños y niñas construyeron durante sus experiencias del día a día y relacionan y emplean al discutir cambios de estado en el aula. Por ejemplo, la relación entre el frío y la aparición de vaho en el aliento. Definimos conocimiento científico escolar como el discutido y construido por el alumnado mediante su participación en el proyecto, por ejemplo, la noción de estado líquido y estado gaseoso. Según Fleer y Pramling (2015), la relación entre ambos es dialógica, no compartimentada y contribuye a la construcción de conceptos en la mente de niños y niñas.

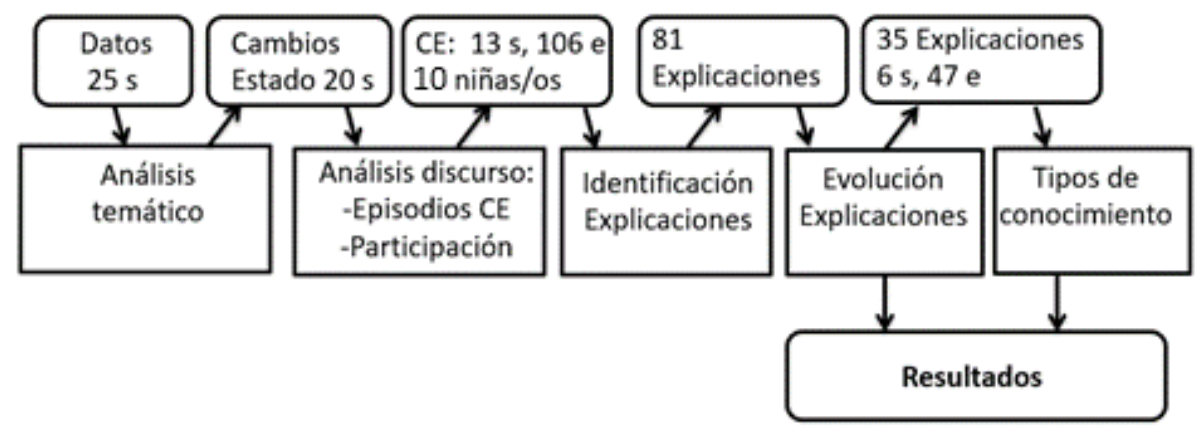

Figura 1. Proceso de reducción y análisis de datos. (CE= cambios de estado; e=episodios; s=sesiones.)

\section{Resultados: características de las explicaciones sobre cambios de estado en tercero de educación infantil}

Se presentan en primer lugar los resultados acerca de los tipos de explicaciones sobre cambios de estado, en cuanto a la identificación de componentes y procesos y al establecimiento de relaciones, identificados en el discurso del alumnado; en segundo lugar, su evolución a lo largo del proyecto.

\section{Tipos de explicaciones sobre cambios de estado en el discurso del alumnado}

La participación en el discurso es desigual. Una niña y nueve niños representan el 52\% de los turnos del alumnado (Tabla 2). Se discuten los resultados de estos 10 niñas y niños cuya participación fue alta y media, ya que no resulta posible identificar pautas en las de los otros 13, cuya participación fue baja. La media individual de los turnos de estos 13 niños representa el $1,5 \%$ de las intervenciones totales en el discurso del aula, con lo cual no es posible establecer una evolución en sus explicaciones orales.

Las explicaciones de estos 10 niños y niñas sobre los cambios de estado se realizaron y evolucionaron, sobre todo en seis sesiones, comprendidas entre la 5 y la 14: 5, 6, 7, 8, 13 y 14. Antes de la sesión 5 no habían empezado a estudiar sistemáticamente el fenómeno, y después de la 14, aunque siguieron discutiendo cambios de estado, no ampliaron sus explicaciones.

La Tabla 3 resume la identificación de componentes y procesos; la construcción de explicaciones y los criterios que cumple cada una, con ejemplos del diálogo del alumnado. La mayoría de las explicaciones se corresponden con relaciones causales (22 turnos). En siete turnos los participantes describen cómo tiene lugar el fenómeno; y en seis aplican una explicación formulada con anterioridad a un nuevo contexto. 
Tabla 3. Discurso del alumnado sobre cambios de estado

\begin{tabular}{|l|c|l|}
\hline Categoría & $\begin{array}{c}\text { Número de } \\
\text { turnos }\end{array}$ & Ejemplo del discurso del alumnado \\
\hline Identificación de componentes & $\mathbf{5 8}$ & Las nubes están hechas de agua \\
\hline Identificación de procesos & $\mathbf{1 0 0}$ & {$[$ El suelo] se va a secar } \\
\hline Explicación & $\mathbf{3 5}$ & \\
\hline Se expresa una relación causal & 22 & {$[$ El agua se va a secar $]$ por el calor que hace } \\
\hline $\begin{array}{l}\text { Se describe como tiene lugar el } \\
\text { fenómeno }\end{array}$ & 7 & Con las gotitas el calor las lleva hasta el espejo \\
\hline $\begin{array}{l}\text { Se aplica una explicación dada } \\
\text { a un contexto nuevo }\end{array}$ & 6 & [En las bolsas no hay gotas] porque están cerradas \\
\hline
\end{tabular}

\section{Evolución en la construcción y uso de explicaciones sobre evaporación y condensación}

La identificación de componentes (agua) y procesos al preparar el experimento 1 en la sesión 5 precede a la construcción de explicaciones (transformación del agua en nubes). Cuatro dimensiones, analizadas a continuación en orden cronológico, tienen particular relevancia en la construcción de explicaciones: la relación causal con el calor; el uso del vocabulario científico, la aplicación de las explicaciones y el vocabulario a contextos cotidianos y la noción de agua en el aire.

Primeras explicaciones: relación causal con el calor y cómo se evaporan las gotas: sesiones 5 y 6

Al final de la sesión 5, la maestra les pregunta sobre el agua que había caído en el suelo durante la preparación del experimento:

Maestra: Entonces, ¿qué pasa con esa agua? [...]

Varios: Se va a secar.

Maestra: ¿Y cómo se va a secar?

Mario: Por el calor que hace.

El alumnado está familiarizado con el proceso de secado, y la relación causal con la temperatura propuesta por Mario está basada en su conocimiento cotidiano.

En la sesión 6 se produjo una evolución notable en las explicaciones del alumnado sobre evaporación a partir de la realización e interpretación de los experimentos 1 y 2 . Al comienzo de la sesión, los niños revisaron las bolsas del experimento 'Evaporación' y discutieron la presencia de gotas de agua en sus paredes internas.

Alberto: [Son] gotas de agua [...] En la bolsa.

Maestra: ¿Dónde están?

Alberto: Arriba del «coso".

Maestra: ¡Anda! Y, ¿cómo puede ser que estén las gotas arriba?

Alberto: ¿Del calor?

$[\ldots]$

Gabriel: Está un poco empañada.

Maestra: Y qué es eso.

Gabriel: Es por el frío.

Los niños reconocen componentes, «gotas de agua», y procesos, «empañado», implicados en los cambios de estado. A continuación, proponen explicaciones y movilizan su conocimiento 
cotidiano sobre la temperatura, estableciendo relaciones causales entre el «frío» y el «calor» y la presencia de gotas de agua, como Mario en la sesión anterior.

Después de examinar las bolsas del experimento 'Evaporación', la maestra introdujo el material del experimento 'Ebullición' y les preguntó qué ocurría en su casa cuando sus padres cocinaban pasta: «Que hace burbujas». Los niños midieron una cantidad de agua y la maestra la puso a calentar en un recipiente abierto. Aplicaron su conocimiento cotidiano para explicar la relación entre la aparición de «humo» y la temperatura del agua: «[Sé que el agua está caliente sin tocar porque] echa humo». A continuación, observaron que el recipiente donde habían calentado el agua estaba vacío y tocaron un espejo que la maestra había situado encima del recipiente, identificando las gotas de agua:

Maestra: ¿Qué hay en el espejo?

Varios: ¡Agua!

$[\ldots]$

Maestra: Cuando yo puse el espejo encima del calentador, ¿'estaba con agua?

Varios: No.

Miguel: A lo mejor es el humo la agua.

Maestra: Mirad lo que pasó aquí dentro ¿Dónde está el agua que estaba aquí dentro?

Varios: Ahí [señalando el espejo]

Maestra: ¿Y cómo llegó el agua hasta aquí?

Hablan muchos a la vez.

Alberto: ¡El calor! ¡Calor!

David: Con las gotitas de agua el calor las lleva hasta el espejo.

En esta conversación, los niños parecen dar por supuesto un proceso de transformación: el agua líquida puede ser transformada en «humo» cuando se calienta. Justifican sus explicaciones en base las siguientes observaciones derivadas de la experimentación: 1) sale humo del recipiente; 2) después de calentar ya no hay agua en el recipiente; y 3) hay agua en el espejo. En el último turno, David propone un mecanismo para explicar cómo se movió el agua, en pequeñas gotitas.

Después de esta experiencia, los niños aceptaron que las gotas de agua podían abandonar el volumen de agua líquida. La maestra indicó que para denominar el proceso se usaba el término evaporación. A continuación, les preguntó de nuevo por las gotas en las bolsas de agua:

Maestra: ¿Ahora ya sabéis qué son estas gotas de agua que hay en las bolsas?

David: Se está evaporando.

Maestra: Se está evaporando... ¿lo qué?

David: ¡Las gotitas! [...]

Miguel: El sol las calienta y después... se evapora...y se hace nube.

Los niños aplican el término evaporación para explicar sus observaciones y proponen una causa, el calor del sol, y cómo tiene lugar. Sin embargo, no discuten la condensación. Miguel, además, apunta a las nubes como el destino final del agua evaporada, lo que es coherente con las predicciones del experimento 'Evaporación'. A continuación, los niños aplicaron sus explicaciones sobre evaporación a las diferencias observadas entre las bolsas y los vasos: «[En los vasos no hay gotas porque] no están cerrados».

Explicaciones sobre evaporación y condensación en contextos cotidianos: sesión 7

En la sesión 7 el alumnado fue capaz de relacionar fenómenos de su vida diaria con el proceso de evaporación, empleando ese término. La sesión comenzó en el patio, donde los niños 
estaban haciendo una "nube" con su aliento en el 'Nuboscopio', una lámina plástica con un agujero en el medio que usaron a lo largo del proyecto para observar el cielo:

Maestra: ¿Que hicimos ahora con el 'Nuboscopio'?

David: Echar nuestro aliento en el 'Nuboscopio'. ¡Que se puso con agua!

Mario: ¡Se evaporó! El agua del aliento.

Maestra. ¿Qué agua se evaporó? Si yo hago así, ¿'hay agua?

Mario: No, solo cuando estás fuera.

$[\ldots]$

Niño: Cuando está frío...

El conocimiento cotidiano («sólo cuando estás fuera») y el conocimiento científico escolar («se evaporó») interaccionan en este fragmento. A continuación, la maestra les preguntó por el experimento 'Ebullición':

Alberto: Que las gotitas se evaporaron y se subieron al espejo.

Maestra: [Repite]. Y, en el espejo, ¿qué pasó? Se quedaron...

Varios: ¡Pegadas!

Maestra: Pero ¿cómo es el aire que echamos de la boca?

No identificado: Caliente.

Maestra: ¿Y el 'Nuboscopio’?

$[\ldots]$

Varios: Frío.

Maestra: ¿Cómo está el espejo?

Varios: Frío.

[La maestra les explica que cuando el agua del aliento encuentra algo frío se enfría y se forman "gotitas" por la diferencia de temperatura.]

Alberto: ¡En la ventana!

Maestra: Y, ¿por qué aquí no me sale? [echa el aliento en el radiador del aula].

Alberto: ¡Porque está caliente!

Al final de la discusión, Alberto es capaz de aplicar la explicación a un nuevo contexto.

Apropiación de vocabulario cientifico sobre los tres estados del agua: sesión 8

En la sesión 8 la maestra introdujo la nomenclatura de los tres estados del agua. Los niños identificaron los tres estados en su vida cotidiana:

Maestra: ¿Qué es el agua?, ¿qué es? ¿Qué es la coca-cola? ¿Qué es el...?

Un niño: ¡Son bebidas!

Alberto: Líquida. [...]

Maestra: Muy bien. Es agua... ¡ilíquida!! ¡Está líquida! ¿Vale, chicos? Su estado es líquido. Cuando está en el congelador... ¿cómo está?

Un niño: Líquida.

Maestra: ¿En el congelador?

Varios: Fría, líquida...

Una niña: ¡Dura!

Maestra: Está dura, no está líquida. Está só-li-da. [...] Cuando la sacamos del grifo o está en una botella, o está así a temperatura normal... está... ¿cómo?

Una niña: Sólida.

Alberto: Líquida.

Maestra: Líquida. Y, y ... cuando el agua se calienta... cando la calentamos... co... ¿¿n qué se convierte esa agua?

Varios: En vapooor. 
Maestra. En vapor de agua [...].El agua puede estar en estado...

$[\ldots]$

Un niño: ¡En estado duro!

Maestra: En estado duro, que es el estado sólido. Muy bien.

Alberto: $\mathrm{Y}$ en estado... caliente.

Maestra: Y en estado... Vapor, que se dice estado "ga-se-o-so".

$\mathrm{Al}$ no resultarles fácil emplear la nomenclatura sobre los tres estados del agua en su vida diaria, los niños propusieron una propia. La maestra legitimó sus intervenciones. A continuación, la maestra intenta que apliquen estos términos para explicar el experimento 'Ebullición':

Maestra: Lo que echamos aquí era... ¿qué?

Varios: Agua.

Maestra: Agua... agua, ¿qué?

Varios: Líquida.

Maestra: Líquida. Cuando le damos calor al agua líquida... [...]

Andrea: Se evapora.

El agua que se evapora puede estar en el aire: sesiones 8,13 y 14

Sesión 8: Para los niños fue fácil explicar la evaporación del agua del recipiente en el experimento 'Ebullición', pero no la evaporación del agua después de condensarse en el espejo. Interpretamos que se puede deber a que el agua del recipiente entra en contacto con el espejo y se condensa en él, lo que es un proceso visible; mientras que la evaporación del espejo al aire no es visible, por lo que es más difícil de explicar:

Maestra: ¿Dónde está el agua que estaba en el espejo?

Javier: Se secó.

Maestra: Se secó, sí, muy bien... pero... ¿dónde está?

Un niño: Se fue a las nubes.

Mucho ruido.

Igor: Está en el aire.

En este fragmento aparece de nuevo la idea de que el agua se va a las nubes cuando se evapora, a no ser que se "pegue" en una superficie. Sólo Igor propone que está en el aire.

En la sesión 13 la maestra introdujo dos analogías para facilitar la visualización de las partículas en el aire, y que los niños pudieran entender la presencia en el aire de gotas de agua no son visibles, idea necesaria para interpretar estos experimentos. La primera consistió en echar colonia en una esquina y les preguntó cómo era posible olerla desde el otro lado:

Maestra: ¿Cómo puede llegar la colonia ahí?

Igor: Porque el aire... [...] La empujó.

Segundo, iluminó las partículas de polvo del aire con el proyector e instó al grupo a imaginar que el "agua en el aire" era similar. En la sesión 14 el grupo retomó la idea de "agua en el aire" al discutir la variación en la altura del agua de los vasos del experimento 'Evaporación'.

Maestra: El agua que se evapora del vaso... que está ahí... esa agua...

David: Está en el viento.

Maestra: No.

Un niño: En el aire.

Maestra: Está en el aire... ¿de dónde?

David y otro niño: De aquí.

Maestra: ¡De esta clase!

David: Es que todo... Fuera, aquí, en todos lados... hay aire. 


\section{Evolución global de las explicaciones sobre cambios de estado}

La evolución se representa en la Figura 2. Se ilustra esta evolución tomando como ejemplo algunas de las explicaciones identificadas en las transcripciones. A izquierda y derecha representamos, respectivamente, explicaciones sobre evaporación y condensación representativas de los pasos que siguió su evolución a lo largo del proyecto conforme a los criterios descritos en la sección de métodos. Las dos columnas centrales muestran los dos tipos de conocimiento movilizados por los niños y niñas para construir sus explicaciones: conocimiento cotidiano y conocimiento científico escolar.

El punto de partida de las explicaciones del alumnado es el reconocimiento de que el agua (componente) puede sufrir transformaciones (procesos), como secarse o transformarse en nubes. A continuación, discutimos las explicaciones sobre cada uno de los procesos.

Las explicaciones sobre evaporación mejoraron sobre todo durante las sesiones 5, 6 y 7, en las que los niños realizaron los experimentos 'Evaporación' y ' Ebullición'. En las sesiones 5 y 6 , basándose en su conocimiento cotidiano, el alumnado construyó explicaciones causales, relacionando el proceso con la temperatura. Segundo, observar la ebullición les proporcionó una forma de explicar cómo las gotas dejen el volumen de agua es el calor y aplicar sus explicaciones a otros contextos (sesión 7). Tercero, en la sesión 8, los niños aprendieron los términos líquido y gas y los aplicaron para explicar el proceso de evaporación, refiriéndose a él como un cambio de líquido a gas. Finalmente, todos salvo uno mostraron dificultades para aceptar que, de alguna manera, podía haber agua en el aire, pero estas dificultades fueron parcialmente superadas después de que la maestra utilizase dos analogías para ayudarles a visualizar de la noción de partículas en el aire en la sesión 13.

En cuanto al fenómeno de condensación, las explicaciones evolucionaron principalmente durante las sesiones 6 y 7. Los niños reconocieron las gotas en las paredes de las bolsas como «empañado» y relacionaron su aparición con el frío y el calor (sesión 6). Lo definieron como gotas que «se pegan» y que se pueden ver sólo cuando hace frío (sesión 7). Sin embargo, no emplearon el término condensación pese a que la maestra lo introdujo en la conversación y tampoco identificaron el proceso como un cambio de estado de gas a líquido, sólo se refirieron al proceso de evaporación como la causa de que las gotas, bien del aliento, bien del recipiente del experimento 2 , se movieran y fueran visibles en otro lugar.

En resumen, los resultados indican que el alumnado fue incluyendo paulatinamente más dimensiones en sus explicaciones sobre evaporación que sobre condensación; y que las explicaciones sobre ambos fenómenos fueron construidas a partir de su conocimiento cotidiano y del conocimiento científico escolar. En esta clase se identificó una clara diferencia entre condensación y evaporación. Fueron capaces de explicar el cómo tiene lugar la evaporación a nivel macro (descenso en el nivel de agua de los vasos) y microscópico (gotitas de agua que van al aire) y se refirieron al proceso como agua líquida que se convierte en vapor. Sin embargo, no se refirieron a la condensación como el proceso inverso, sino como gotas que «se pegan». Es decir, sólo llegaron a proponer una explicación sobre cómo tiene lugar este proceso desde el punto de vista macroscópico. En consecuencia, fueron capaces de conectar más situaciones y conocimientos de su vida diaria con el fenómeno de evaporación que con el de condensación (ver Figura 2). Además, aunque aplicaron sus explicaciones sobre evaporación a todos los contextos con los que tuvieron contacto, no hicieron lo mismo con las explicaciones sobre condensación. 


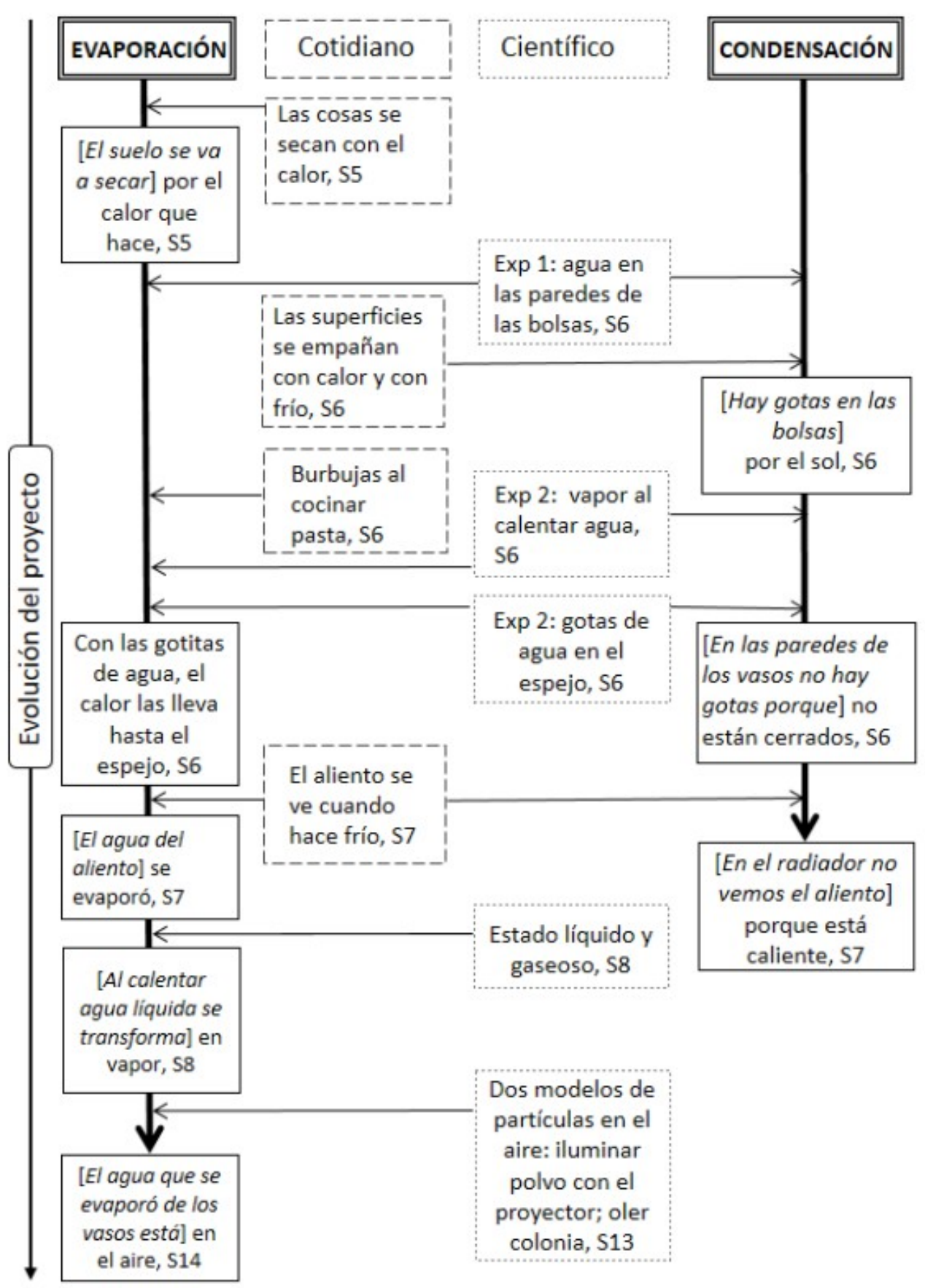

Figura 2. Evolución de las explicaciones sobre evaporación y condensación a lo largo del proyecto (sesión: S-n). Leyenda: Exp 1, experimento 'Evaporación', Exp 2, experimento 'Ebullición'.

\section{Discusión}

El objetivo del trabajo es examinar cómo explican niños y niñas los cambios de estado presentes en su vida y en los experimentos propuestos en el aula. Identificamos los pasos que siguen en su construcción de manera que estas se vuelven progresivamente más complejas y qué tipos de conocimiento emplean para ello. El punto de partida para construir explicaciones fue la identificación de componentes, seguido de la identificación de procesos. Las explicaciones causales son las primeras, y en ellas niños y niñas relacionan los fenómenos con 
sus experiencias cotidianas. Las explicaciones sobre cómo los procesos tienen lugar, son las siguientes en aparecer. Creemos que estas requieren un mayor conocimiento del fenómeno, y en nuestro estudio, una vez son formuladas, el alumnado identifica los procesos en una mayor variedad de contextos.

El alumnado en nuestro estudio presentó mayor facilidad para explicar y comprender la evaporación que la condensación. El paso inicial fue construir una explicación causal sobre la relación entre este proceso y el calor, y el final la apropiación de la idea de que puede haber agua en el aire. En cuanto a la condensación, aunque identificaron en diferentes contextos las gotas condensadas como agua y que sólo eran observables cuando la temperatura era baja, no reconocieron que implicaba un cambio de estado. Creemos que se debería prestar más atención a la transformación gas-líquido para permitir que estas explicaciones evolucionen. El experimento 2, sobre ebullición, fue de gran ayuda para identificar que el 'humo' y el agua del recipiente eran la misma substancia, pero el proceso de condensación no fue discutido como cambio y por ello no resultó útil para comprenderlo. Además, tampoco resulta indicado para comprender la formación de nubes, punto de partida del proyecto.

Creemos conveniente comenzar a estudiar cambios de estado en educación infantil porque están presentes en la vida diaria de niñas y niños. Al trabajarlos, pueden relacionar sus experiencias cotidianas con las del aula y mejorar su comprensión. Las explicaciones que construyen no incluyen todas las interrelaciones, pero pueden servirles de base sobre la que desarrollar otras más complejas más adelante. Aunque son capaces de aprender vocabulario sobre cambios de estado y usarlo en sus explicaciones, en nuestro estudio y otros sobre alumnado de mayor edad (e. g. Johnson, 1998) términos como aire y viento; y humo y vapor, fueron usados indistintamente, por lo que su uso se debe examinar para saber a qué se refieren. El alumnado de este estudio sólo empleó términos sobre los que construyó significados: es decir, mientras que usaron el término evaporación de forma apropiada y en diferentes contextos, no lo hicieron con el término condensación, pese a los esfuerzos de la maestra. Sugerimos que el estudio de estos fenómenos en educación infantil debe estar andamiado. Aunque no es el núcleo de nuestro análisis, las trascripciones indican que, con sus intervenciones en el discurso, en todo momento, la maestra empleó estrategias de andamiaje: reclamó atención y reflexión sobre el fenómeno bajo estudio (Pramling Samuelsson y Asplund Carlsson, 2008) y promovió la creación de espacios compartidos (Gustavsson, Jonsson, LjungDjärf y Thulin, 2016).

La repetición a lo largo del proyecto de los experimentos y las oportunidades para discutir y reflexionar sobre ellos favorecieron la modificación de explicaciones conforme a las nuevas experiencias y su aplicación a otros contextos. Por ello, sugerimos tener en cuenta la recurrencia en el diseño curricular, ya que favorece el aprendizaje y posibilita la revisión de modelos explicativos. Además, proporcionar al alumnado tiempo y espacio para la discusión favorece la construcción colectiva de explicaciones.

Los cambios de estado implican procesos y entidades no observables, lo que supone dificultades para el alumnado de menor edad, por la importancia de la percepción con los sentidos (Piaget, 1947). Recomendamos la realización de experiencias perceptibles con los sentidos. En esta clase las explicaciones sobre evaporación mejoraron una vez que observaron el volumen de vapor saliendo del recipiente caliente y tocaron las gotas de agua en el espejo. Además, les resultó más fácil explicar que el agua al evaporarse acaba en el espejo (visible) que en el aire (no visible). Son resultados consistentes con los de Tytler (2000), quien indica que el alumnado de 6-7 años tenía más dificultades para emplear la noción de «agua en el aire» que el de 12-14. 
En cuanto a las limitaciones del estudio, en nuestro análisis nos centramos en los "porqués" y "cómo" de las explicaciones y en su aplicación a nuevos contextos y en los tipos de conocimientos movilizados. Debido a las limitaciones de espacio no abordamos otros factores relevantes en el aprendizaje, como los afectivos (Siry, 2014) o las estrategias de la maestra (Gustavsson et al., 2016). Por otro lado, debido a nuestra perspectiva en la que las conversaciones colectivas son las que construyen el aprendizaje en un aula (Mercer, 2000), no identificamos trayectorias individuales. Cada persona aprende de manera diferente y un análisis individual nos brindaría información sobre las formas de aproximarse a estos fenómenos y a la construcción de explicaciones. Por ejemplo, Tytler y Peterson (2004) estudiaron en profundidad las intervenciones de un niño y una niña a lo largo de su estudio longitudinal, lo que les permitió examinar diferencias en su forma de aprender, pese a que ambos habían alcanzado a priori el mismo nivel de comprensión. Un buen dominio del lenguaje y el querer complacer a los adultos, llevaron a que la niña emplease con mayor frecuencia términos técnicos como evaporación. El niño, por otro lado, mostró tendencia a recordar principalmente ideas y conceptos, en contraposición a su compañera que recordaba con mayor claridad las escenas e interacciones con otros en la realización de los experimentos.

\section{Agradecimientos}

A la maestra, las niñas y niños. Al Ministerio de Economía y Competitividad (MINECO), que financió tanto el contrato de formación predoctoral de Sabela F. Monteira, código BES-2013-062873 (asociado al proyecto EDU2012-38022-C02-01); como el proyecto EDU2015-6643-C2-2-P.

\section{Referencias}

Berland L. K., McNeill, K. L. (2010) A learning progression for scientific argumentation: Understanding student work and designing supportive instructional contexts. Science Education 94(5) 765-793.

Cruz-Guzmán M., García-Carmona A., Criado A. M. (2017) Aprendiendo sobre los cambios de estado en educación infantil mediante secuencias de pregunta-predicción comprobación experimental. Enseñanza de las Ciencias, 35 (3), 175-193. doi: $10.5565 / \mathrm{rev} /$ ensciencias. 2336

Fleer M., Pramling N. (2015) A cultural-bistorical study of cbildren learning science: Foregrounding affective imagination in play-based settings.Dordrecht, The Netherlands: Springer

Gee J. P. (2005) An introduction to discourse analysis: Theory and method. London: Routlegde.

Gustavsson L., Jonsson A., Ljung-Djärf A., Thulin, S. (2016) Ways of dealing with science learning: a study based on Swedish early childhood education practice. International Journal of Science Education, 38 (11), 1867-1881, doi: 10.1080/09500693.2016.1220650

Jiménez Aleixandre M. P. (2010) 10 ideas clave: en argumentación y uso de pruebas. Barcelona: Graó.

Johnson P. M. (1998) Children's understanding of changes of state involving the gas state, Part 1: Boiling water and the particle theory. International Journal of Science Education, 20, 567-583.

Kelly, G. J. \& Green, J. (2019). Orientating ways of thinking: Theory and methods for the study of education. In G. J. Kelly \& J. Green (Eds.) Theory and Methods for Sociocultural Research in Science and Engineering Education. London: Routledge.

Kuhn D., Pearsall S. (2000) Developmental origins of scientific thinking. Journal of Cognition and Development, 1, 113-129.

Littleton, K. \& Mercer, N. (2013) Interthinking: putting talk to work. Abingdon: Routledge. 
Leuchter M., Saalbach H., Hardy I. (2014) Designing Science Learning in the First Years of Schooling. An intervention study with sequenced learning material on the topic of 'floating and sinking'. International Journal of Science Education, 36 (10), 1751-1771. doi:10.1080/09500693.2013.878482.

Neuendorf K. A. (2002) The content analysis guidebook. California: Thousand Oaks.

McNeill K. L. (2011) Elementary students' views on explanation, argumentation and evidence, and their abilities to construct arguments over the school year. Journal of Research in Science Teaching, 48(7), 793-823. doi:10.1002/tea.20430.

McNeill K. L, Krajcik J. (2008) Scientific explanations: Characterizing and evaluating the effects of teachers' instructional practices on student learning. Journal of Research in Science Teaching, 45(1), 53-78. doi: 10.1002/tea.20201.

Mercer, N. (2000) Words and Minds. London: Routledge.

Merriam S. (2009) Qualitative research: A guide to design and implementation. San Francisco, CA: Jossey-Bass.

National Research Council (2012) A Framework for K-12 Science Education: Practices, crosscutting concepts, and core ideas. Washington, DC: The National Academies Press.

Organización para la Cooperación y el Desarrollo Económico (2012) Access to Early Childhood Education. In Education at a glance, 2012: Highlights. Paris: OECD Publishing. Recuperado de: http://dx.doi.org/10.1787/eag_highlights-2012-30-en.

Organización para la Cooperación y el Desarrollo Económico (2016) PISA 2015 Assessment and Analytical Framework. Science, Reading, Mathematic and Financial Literacy Recuperado de: http://dx.doi.org/10.1787/19963777

Osborne J. (2014) Scientific practices and inquiry in the science classroom, pp. 1835-1901 en Lederman N. G., Abell S. K. (coords.). Handbook of Research on Science Education, Volume II). New York: Routledge.

Piaget J. (1947) The Psychology of Intelligence. London: Routledge.

Pramling Samuelsson I., Asplund Carlsson M. (2008) The playing learning child: Towards a pedagogy of early childhood. Scandinavian Journal of Educational Research 52(6), 623-641. doi:10.1080/00313830802497265.

Siry C., Kremer I. (2011). Children Explain the Rainbow: Using Young Children's Ideas to Guide Science Curricula. Journal of Science Education and Technology, 20(5), 643-655

Siry C., Max C. (2013) The collective construction of a science unit: Framing curricula as emergent from kindergarteners' wondering. Science Education, 97, 878-902. doi: $10.1002 /$ sce. 21076

Siry C. (2014) Towards multidimensional approaches to early childhood science education. Cultural Studies of Science Education, 9(2), 297-304. doi:10.1007/s11422-012-9445-8

Songer N. B., Gotwals A. W. (2012) Guiding explanation construction by children at the entry points of learning progressions. Journal of Research in Science Teaching, 49(2), 141-165. doi: $10.1002 /$ tea.20454

Tytler R. (2000) A comparison of year 1 and year 6 students conceptions of evaporation and condensation: dimensions of conceptual progression. International Journal of Science Education 22 (5), 447 - 467. 
Tytler R., Peterson S. (2004) Young children learning about evaporation: a longitudinal perspective. Canadian journal of science, mathematics and technology information 4 (1), 111-126

Zangori, L.; Forbes, C. T.; \& Schwarz, C. (2015) Exploring the Effect of Embedded Scaffolding Within Curricular Tasks on Third-Grade Students' Model-Based Explanations about Hydrologic Cycling. Science \& Education, 24, 957-981. doi:10.1007/s11191-015-9771-9. 